\title{
Oliver Sartor Talks with Thomas A. Hope, Jeremie Calais, and Wolfgang P. Fendler About FDA Approval of PSMA
}

\author{
Oliver Sartor ${ }^{1}$, Thomas A. Hope ${ }^{2}$, Jeremie Calais ${ }^{3}$, and Wolfgang P. Fendler ${ }^{4}$
}

${ }^{1}$ Tulane University School of Medicine, New Orleans, Louisiana; ${ }^{2}$ Department of Radiology and Biomedical Imaging, University of California, San Francisco, San Francisco, California; ${ }^{3}$ Ahmanson Translational Theranostics Division, Department of Molecular and Medical Pharmacology, David Geffen School of Medicine, UCLA, Los Angeles, California; and ${ }^{4}$ Department of Nuclear Medicine, University of Duisburg-Essen and German Cancer Consortium (DKTK)-University Hospital Essen, Essen, Germany

$\mathbf{O}$

n December 1, the U.S. Food and Drug Administration (FDA) approved ${ }^{68} \mathrm{Ga}$-PSMA-11 for PET imaging of prostate-specific membrane antigen (PSMA)-positive lesions in men with prostate cancer. It is the first approval for a PSMA PET

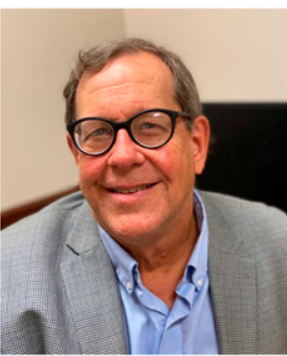

Oliver Sartor, MD

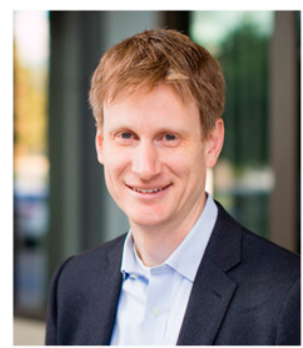

Thomas A. Hope, MD tracer in the United States. The approval was granted to the University of California Los Angeles (UCLA) and the University of California San Francisco (UCSF), which filed New Drug Applications (NDAs) based on clinical trial results and published data. Oliver Sartor, MD, discussed the NDA process and prospects for PSMA imaging with Thomas Hope, $\mathrm{MD}$, associate professor-in-residence and Director of Molecular Therapy at UCSF; Jeremie Calais, MD, MSc, assistant professor and Director of the Clinical Research Program in Nuclear Medicine and Theranostics at UCLA; and Wolfgang Fendler, MD, former UCLA investigator and now Vice Chair of Nuclear Medicine at the Essen University Hospital (Germany). Dr. Sartor is an internationally recognized expert in prostate cancer. He is the Assistant Dean for Oncology, Medical Director of the Tulane Cancer Center, and the Laborde Professor for Cancer Research at Tulane Medical School, with appointments in both Medicine and Urology. He has published more than 400 peer-reviewed articles and led or co-led multiple national and international clinical studies, including 3 phase III studies pivotal for FDA approval. He has lectured in more than 30 countries. He is a past member of the National Cancer Institute Board of Scientific Counselors (Clinical

Sciences and Epidemiology) and is coPI of the VISION trial, an international phase III study of ${ }^{177} \mathrm{Lu}-\mathrm{PSMA}$ in advanced prostate cancer.

Dr. Sartor: It's really exciting to be able to have an FDA approval on PSMA PET. I'd like to hear about the process and the application to the FDA. What made you think that an academic institution could create a successful application?

Dr. Hope: Back in 2015, DOTATATE was just being submitted for neuroendocrine tumors as an NDA, and the data for DOTATOC had been acquired from the University of Iowa. I had seen the process they were going through and realized that this was something an academic group could actually do. Perhaps more important, Louis Marzella, MD, $\mathrm{PhD}$, from the FDA, publicly stated that he supported these types of NDAs and wanted to see

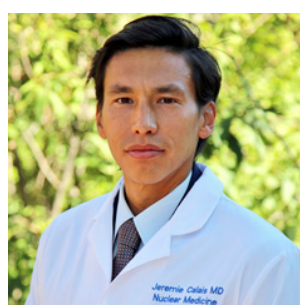
Jeremie Calais, MD, MSc

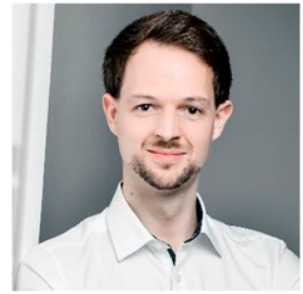

Wolfgang P. Fendler, MD academic groups get drugs approved. So, the combination of experience with neuroendocrine tumors and the support and excitement from the FDA made us believe we could do this. I would also specifically point to John Sunderland, PhD, MBA, from Iowa, without whose advice and support this project would never have been possible.

Dr. Sartor: I'd like to hear a little bit about the process of this application.

Dr. Hope: In all honesty, what was most exciting about this process was the fact that UCLA and UCSF were able to truly collaborate. There is no lead. The FDA allowed us to submit 2 NDAs on the same day with 2 identical PDUFA [Prescription Drug User Fee Act] dates. Never before has there been a drug with 2

We would like to thank all the patients and their referring physicians whose willingness to participate made these studies possible. We thank U.S. and German investigators for data sharing and outstanding academic support. We would also like to thank the entire UCLA and UCSF clinical faculty and research staff whose hard work enabled the successful NDA, especially Joseph Blecha, Giuseppe Carlucci, Johannes Czernin, Matthias Eiber, Ken Herrmann, Robin Ippisch, Ashley Mishoe, Roger Slavik, Sara St. James, John Sunderland, and Shaojun Zhu. — Thomas A. Hope, Jeremie Calais, and Wolfgang P. Fendler

COPYRIGHT (c) 2021 by the Society of Nuclear Medicine and Molecular Imaging.

DOI: $10.2967 /$ jnumed.120.261834 
manufacturers approved at the same time, and the fact the FDA let us submit 2 was central to encouraging this type of collaboration.

Dr. Sartor: Tell us about the label and how you anticipate this label will be incorporated into practice.

Dr. Hope: In terms of the label, I would say we were very fortunate, as there was a lot of debate as to whether or not the FDA would acknowledge our initial staging data. There was hesitation because we did not hit our primary endpoint in our initial staging study. We ended up with sensitivity around $40 \%$, and we were planning for a sensitivity in the high $60 \%$ range. We were grateful that the FDA acknowledged that even though the sensitivity was lower, there was still significant clinical impact.

Our label ended up saying that PSMA-11 is indicated for detecting PSMA-positive lesions in prostate cancer patients with suspected metastasis who are candidates for initial definitive therapy and patients with suspected recurrence based on elevated serum prostatespecific antigen (PSA) levels. We ended up in a very good place, where the FDA allowed both initial staging and biochemical recurrence on the label. This will be very important, because it will be the first PET radiotracer indicated in initial staging. This will now help convince (actually force) Medicare to also include coverage for PET imaging at initial staging of prostate cancer.

Dr. Sartor: What endpoints were critical for the FDA? And how did you work with the FDA to craft those endpoints?

Dr. Fendler: The 2 trials assessed ${ }^{68} \mathrm{Ga}$-PSMA-11 in the settings of biochemical recurrence and initial staging. In the biochemical recurrence trial, the primary endpoint was positive predictive value for localization of disease. In the initial staging trial, the primary
Dr. Fendler: Overall, it is important to have an accurate imaging test, which can be achieved with both ${ }^{68} \mathrm{Ga}$ and ${ }^{18} \mathrm{~F}$ compounds. There are minor technical differences. ${ }^{18} \mathrm{~F}$ has a longer half-life and may be produced centrally at fewer cyclotron sites with nationwide shipment. On the other hand, nonspecific bone uptake has been described for some of the ${ }^{18} \mathrm{~F}$ compounds, which makes interpretation of bone findings more challenging. ${ }^{68} \mathrm{Ga}$ has a shorter half-life and must be produced in closer-by facilities or radiopharmacies with a gallium generator, similar to the approved ${ }^{68} \mathrm{Ga}$-DOTATATE. Fast and easy local production certainly contributed to widespread use of ${ }^{68}$ Ga-PSMA-11 and assessment in clinical trials such as our Investigational New Drug (IND) studies.

Dr. Sartor: Will there be any restrictions on ${ }^{68}$ Ga-PSMA PET with regard to sites that have access to this technology?

Dr. Hope: Similar to ${ }^{11} \mathrm{C}$-choline, approved at Mayo Clinic only, with our approval, the PSMA-11 will be available only at UCSF and UCLA. But there is an important distinction from ${ }^{11} \mathrm{C}$ choline: a company (and we anticipate that multiple companies will do this) can submit an Abbreviated New Drug Application (ANDA) for a kit. Once approved, a kit can then be used at any site without having to go through a site-specific ANDA process. So, the path to get widespread availability is subsequent approval of a kit to make this compound based on our NDA. A second approach would be for radiopharmacies to submit their own ANDAs. But for the first 18 months, it will be available only at UCSF and UCLA.

Dr. Fendler: Kit-based production and availability has already been achieved for ${ }^{68} \mathrm{Ga}$-DOTATATE on a large scale. This will be similarly doable for ${ }^{68} \mathrm{Ga}$-PSMA-11 once there is alternative availability.

"We ended up in a very good place, where the FDA allowed both initial staging and biochemical recurrence on the label."

endpoint was sensitivity and specificity for detection of pelvic nodal metastases. An important aspect was validation of the ${ }^{68} \mathrm{Ga}-\mathrm{PSMA}-$ 11 PET findings by an independent method such as histopathology or follow-up imaging. An independent validation framework was important for us and the FDA to assess the true meaning of PET uptake by a ground truth, whenever available. Both trials had key secondary endpoints to assess clinical impact and robustness, such as change in management or reproducibility of ${ }^{68}$ Ga-PSMA-11 PET findings.

Dr. Hope: To add one more detail, in the biochemical recurrence trial, the FDA initially and historically has always wanted pathology correlation, and in this population we are imaging patients with very low PSAs, where their lesions often cannot be biopsied. The discussion with the FDA focused on how to craft a composite endpoint that combined both imaging and clinical follow-up with pathology. We spent quite a lot of time to convince the FDA that if you stuck with pathology, you would miss the clinically relevant patients.

Dr. Sartor: One question that has arisen is the comparability between the isotopes ${ }^{68} \mathrm{Ga}$ and ${ }^{18} \mathrm{~F}$. Could you comment on what might be some advantages or disadvantages for each isotope?

Dr. Calais: I think most of the ${ }^{68} \mathrm{Ga}$ - and ${ }^{18} \mathrm{~F}$-PSMA PET probes advanced in their clinical development (e.g., ${ }^{68}$ Ga-PSMA-11 and ${ }^{18} \mathrm{~F}-\mathrm{DCFPyL}$ ) perform very well for prostate cancer lesion detection. There are some physical technical differences that do not really translate into clinically significant differences in terms of diagnostic efficacy. What matters is to have the patient getting a PSMA PET scan. So, at the end, it is the local availability of the PSMA PET tracer that really matters clinically, not the choice of ${ }^{68} \mathrm{Ga}$ or ${ }^{18} \mathrm{~F}$.
Dr. Calais: I can also add that both the UCLA and the UCSF NDAs use conventional radiolabeling and are not cold-kit based. UCLA and UCSF NDAs waived market exclusivity, which enables any academic site in the United States that has the ability to manufacture ${ }^{68} \mathrm{Ga}-\mathrm{PSMA}-11$, even without the kit-based method, similar to the UCLA/UCSF NDAs, to file ANDAs.

Dr. Sartor: We have targets and isotopes, but we have not talked much about the PSMA-binding molecule itself. Could you comment on the ligand that was used within the application and how you see other molecules involved in this space?

Dr. Calais: The 2 main unpatented ${ }^{68} \mathrm{Ga}$-PSMA PET ligands are PSMA-11 and PSMA I\&T.

Dr. Sartor: Which was in the application?

Dr. Calais: PSMA-11, for multiple historic reasons. The first human experience with PSMA PET imaging for prostate cancer was reported in 2012 by the team at the University of Heidelberg [Afshar-Oromieh et al. Eur J Nucl Med Mol Imaging. 2012;39(6): 1085-1086], and they used ${ }^{68}$ Ga-PSMA-11. Most of the literature and published data were then with PSMA-11. As a reminder, there was and is no protected patent, so basically any site that can perform ${ }^{68} \mathrm{Ga}$ radiolabeling was able to do it. In our NDA and in many other institutions, the easiest way was to use already published data on PSMA-11 pharmacology, dosimetry, or any efficacy endpoint for diagnostic accuracy that was already published. PSMA-11 is the PSMA tracer with the most published data. There might have been a snowball effect with more PSMA-11 published data and more new sites using PSMA-11 instead of other tracers, such as PSMA I\&T. 
Another reason is that PSMA-11 was the smallest in size, and usually smaller molecules tend to have a faster clearance time from plasma to tumor targets, which is suitable for early imaging time points, such as with ${ }^{68} \mathrm{Ga}$.

Dr. Hope: Two quick comments. First, when we talk about the ligand, we are talking about the urea motif, the binding molecule that targets PSMA. It is really important to note that all these molecules except for one, the CTT family of compounds, have the same binding moiety to the PSMA molecule. So just keep in mind that I\&T, DCFPYL, PSMA-11-all these different compounds bind to PSMA using the exact same molecule. So, it is really just the back end of the molecule that varies across them.

Second, as to why we use PSMA-11. Back when we started working with PSMA-11, there was no I\&T or many of these other compounds. PSMA-11 was the only nonpatented compound. I have to give a lot of acknowledgment to the University of Heidelberg and Ali Afshar-Oromieh, MD. He was very kind and lent us dosimetry and toxicity data. Without that, we would not have been able to have an IND. PSMA-11 was the first compound that was not patented, and Heidelberg was incredibly collaborative and open.

Dr. Sartor: Many people were concerned about sensitivity, even though this scan is the best scan ever invented. What do you regard as the limitations to sensitivity in the recurrent setting? Do you have PSA cutoffs that you might recommend being followed for clinicians who will order the test?

Dr. Fendler: In the recurrence setting, PSA is a good indicator of detectable tumor burden. PSA measurement is widely available and included in clinical guidelines and workflows. In patients with recurrence after prostatectomy, a PSA of $0.2 \mathrm{ng} / \mathrm{mL}$ is a reasonable cutoff for PSMA PET assessment. At PSA levels below this cutoff, the probability of tumor detection is lower than $30 \%$. For PSA $>0.5 \mathrm{ng} / \mathrm{mL}$, the probability of tumor detection is $>50 \%$. However, PET may be performed earlier or independent from PSA levels if there are additional risk factors, in cases of planned local therapy, or in a PSA-negative or -suppressed setting. PSA offers good guidance for the use of PSMA PET, but in the end it is an individual decision.

Dr. Sartor: If someone starts androgen deprivation therapy (ADT), what might be the effects on PSMA expression? And can you comment specifically on the kinetics of change after starting ADT?

Dr. Calais: Let's start with the castrate-sensitive setting. Here I think it is pretty linear. After administration of first-line ADT, the size of tumor lesions will decrease, with some cell killing. Your PSMA PET signal will then decrease as well, indicating response to first-line ADT. In the castrate-sensitive setting, after 2 months of ADT fewer lesions or less uptake would be visible in the same regions on a follow-up PSMA PET scan. That would indicate a response. Then if the patient becomes castrate resistant, you would see under the same ADT line some increasing PSMA uptake on new lesions on the PSMA PET scan.

With castrate-resistant disease under second-generation androgen receptor-signaling inhibitor agents it gets a little bit trickier. Androgen receptor signaling and PSMA expression are linked. Many people want to try to use these to modulate PSMA expression to increase the sensitivity of PSMA PET or the efficacy of PSMAtargeted therapy. This seems to have been possible in some patients and some lesions, but not very reproducible. There is a very high heterogeneity of effects of these molecules on the PSMA signaling, both per lesion and per patient. So, it would be hard to give any guidelines on how to use it before a scan or any new PSMA-targeted therapies.

Dr. Sartor: Let us discuss briefly reasons for a false-positive PSMA scan that is not prostate cancer.

Dr. Calais: I think the trickiest area is the ribs, which are subject to many minor traumas and can exhibit some focal reactional uptake. This, by the way, can happen with many other nuclear medicine tracers and also with PSMA. Sometimes it's tricky. For example, the scan shows a focal faint rib uptake and the patient is not known to be metastatic M1 and on the CT you do not have the sclerosis that you would like to have to call a metastasis nor a fracture. You have nothing, except a focal faint uptake. When there is nothing elsewhere the impact on management is significant, switching the patient from M0 to M1. In these tricky cases, it is very important to take into consideration all the other prostate cancer parameters and pretest positivity likelihood before making a final call. Often the only way to get real truth at the end is on follow-up. Six months later, the PSMA uptake disappeared or you have something on the CT, then you have your call. Usually, when the rib uptake is isolated and faint it is actually not a metastasis. That is the main tricky pitfall I've seen. Then you have all the commonly reported pitfalls seen with any inflammatory or infectious process or the ganglia.

Dr. Hope: All right. I want to ask you a question, Oliver. You've used these tracers. What would you say is the difference between PSMA and fluciclovine?

Dr. Sartor: There's no question about the sensitivity of PSMA as compared with fluciclovine. There's no comparison in terms of sensitivity. In my experience, the PSMA PET is substantially more sensitive than the fluciclovine, especially with PSA levels $<1.0 \mathrm{ng} / \mathrm{mL}$.

Dr. Hope: How quickly was that apparent after you started using the radiotracer?

Dr. Sartor: We quickly found that fluciclovine was less sensitive than what I regarded as optimal, after only a few patients. PSA recurrence is clear, but many patients with PSA in the $0.5-2.0 \mathrm{ng} / \mathrm{mL}$ range had negative fluciclovine, whereas PSMA PET was positive.

Dr. Fendler: How do you think PSMA PET approval will impact clinical guidelines and its use in clinical practice?

Dr. Sartor: I anticipate that guidelines will rapidly adapt to the FDA approval of PSMA PET imaging, given the consequences of detection of disease outside the prostate. I can imagine that in both the upfront and the recurrent settings there will be substantial changes in clinical practice, which will need to be further studied in order to understand their full impact. 